\title{
THE NEAR-RING OF AFFINE TRANSFORMATIONS
}

D. W. BLACKETT

An affine transformation of a vector space $V$ with scalar field $F$ is defined by

$$
\alpha A=\alpha T+\beta
$$

where $\alpha$ is variable in $V, \beta$ is fixed in $V$, and $T$ is a linear transformation of $V$. The affine transformation is nonsingular if and only if $T$ is nonsingular.

A few familiar facts [1] are:

1. The nonsingular affine transformations form the "affine group" under iteration.

2. The translations, i.e. those $A$ with $T=\mathrm{I}$, form a normal subgroup of the affine group.

3. The quotient group of the affine group modulo the translation group is isomorphic to the group of nonsingular linear transformations.

4. If $V$ is finite-dimensional with basis $\left(\alpha_{1}, \cdots, \alpha_{n}\right)$, let

$$
M=\left(\begin{array}{ccc}
m_{11} & \cdots & m_{1 n} \\
\vdots & & \vdots \\
m_{n 1} & \cdots & m_{n n}
\end{array}\right)
$$

be the matrix of $T$ and $B=\left(b_{1}, \cdots, b_{n}\right)$ the coordinate $n$-tuple of $\beta$ relative to the given basis. The correspondence

$$
A \rightarrow\left(\begin{array}{cc}
M & \theta^{\prime} \\
B & 1
\end{array}\right) \quad(\theta=(0, \cdots, 0))
$$

is an isomorphism of the affine group onto a multiplicative group of $(n+1) \times(n+1)$ matrices.

Addition of transformations is not mentioned in 1-4. This is in contrast to theorems about linear transformations such as, "The linear transformations form a simple ring." One reason why addition of affine transformations has been avoided is that affine transformations do not form a ring but only a near-ring $[2 ; 3]$.

The following unfamiliar facts about this near-ring are analogues of 1-4:

Presented to the Society, September 2, 1955; received by the editors June 16, 1955. 
I. The affine transformations (singular ones included) form a nearring with commutative addition.

II. The constant transformations, i.e. those $A$ with $T=0$, form the unique maximal two-sided ideal of the near-ring of affine transformations.

III. The quotient near-ring of affine transformations modulo constant transformations is isomorphic to the simple ring of linear transformations.

IV. If $V$ is finite-dimensional with basis $\left(\alpha_{1}, \cdots, \alpha_{n}\right)$, let $M$ be the matrix of $T$ and $B$ the coordinate $n$-tuple of $\beta$ with respect to the given basis. The correspondence

$$
A \rightarrow\left(\begin{array}{ll}
M & \theta^{\prime} \\
B & e
\end{array}\right)
$$

is an isomorphism onto a near-ring of $(n+1) \times(n+1)$ matrices. The nondistributive element $e$ adjoined to $F$ satisfies the following laws:

$$
\begin{aligned}
& e e=e, \quad e+e=e, \\
& f e=e f=f \quad \text { for all } f \text { in } F .
\end{aligned}
$$

A near-ring is an algebraic system which satisfies all the usual ring axioms except possibly one distributive law and the commutative law of addition. The proof that the linear transformations of a vector space over a field satisfy the ring axioms can be translated into a proof that the affine transformations of the vector space satisfy all of these axioms except the right distributive law. (The proof of the left distributive law uses only the definition of the addition of transformations, while that of the right distributive law uses linearity.) I follows. That the affine transformations are not a ring may be proved: Let $A$ be any affine transformation and $C$ an affine transformation with $T=0$ and $\beta \neq 0$. Then

$$
\alpha(A+A) C=\beta \text { but } \alpha(A C+A C)=\beta+\beta \neq \beta .
$$

A set $S$ of elements of a near-ring $N$ is a two-sided ideal [2] if

a. $S$ is an additive normal subgroup of $N$;

b. $N S \subset S$;

c. $\left(n_{1}+s\right) n_{2}-n_{1} n_{2}$ is in $S$ for all $n_{1}, n_{2}$ in $N$ and $s$ in $S$.

If $N$ is the near-ring of affine transformations and $S$ the set of constant transformations, a and b follow immediately. Let $A_{1}, A_{2}$, and $C$ be affine transformations defined by

$$
\alpha A_{1}=\alpha T_{1}+\beta_{1}, \quad \alpha A_{2}=\alpha T_{2}+\beta_{2}, \quad \alpha C=\beta .
$$

Then 


$$
\begin{aligned}
\alpha\left[\left(A_{1}+C\right) A_{2}-A_{1} A_{2}\right] & =\left(\alpha C+\alpha A_{1}\right) A_{2}-\alpha A_{1} A_{2} \\
& =\left[\left(\beta+\alpha A_{1}\right) T_{2}+\beta_{2}\right]-\left[\alpha A_{1} T_{2}+\beta_{2}\right] \\
& =\beta T_{2}+\alpha A_{1} T_{2}-\alpha A_{1} T_{2}=\beta T_{2} .
\end{aligned}
$$

This shows that the constant transformations are a two-sided ideal of the affine transformations.

Since two affine transformations $A_{1}$ and $A_{2}$ are in the same residue class modulo the constant transformations if and only if $T_{1}=T_{2}$, III is obvious. Because the image of a two-sided ideal of a near-ring under a near-ring homomorphism is a two-sided ideal, the simplicity of the ring of linear transformations implies by III that every twosided ideal of the affine transformations is contained in the ideal of constant transformations. This completes the proof of II. IV follows by direct computation.

\section{REFERENCES}

1. G. Birkhoff and S. MacLane, $A$ survey of modern algebra, rev. ed., New York, Macmillan, 1953, pp. 252-254.

2. D. W. Blackett, Simple and semisimple near-rings, Proc. Amer. Math. Soc. vol. 4 (1953) pp. 772-785.

3. W. E. Deskins, $A$ radical for near-rings, Proc. Amer. Math. Soc. vol. 5 (1954) pp. 825-827.

Boston UnIversity 